\title{
Correction to: A reversible data hiding in encrypted image based on prediction-error estimation and location map
}

Asad Malik $^{1} \cdot$ Hong-Xia Wang ${ }^{2} \cdot$ Yanli Chen ${ }^{1} \cdot$ Ahmad Neyaz Khan $^{3}$

Published online: 25 January 2020

C) Springer Science+Business Media, LLC, part of Springer Nature 2020

\section{Correction to: Multimedia Tools Applications (2019) https://doi.org/10.1007/s11042-019-08460-w}

In the original publication, figure 2 has some incorrect notations and Table 1 has inappropriate alignment. The original article has been corrected.

Publisher's note Springer Nature remains neutral with regard to jurisdictional claims in published maps and institutional affiliations.

The online version of the original article can be found at https://doi.org/10.1007/s11042-019-08460-w

Hong-Xia Wang

hxwang@scu.edu.cn

Asad Malik

asad@my.swjtu.edu.cn

Yanli Chen

yanli027@163.com

Ahmad Neyaz Khan

ahmadnk@std.uestc.edu.cn

1 School of Information Science \& Technology, Southwest Jiaotong University, Chengdu 611756 Sichuan, China

2 College of Cybersecurity, Sichuan University, Chengdu 610065, China

3 University of Electronic Science \& Technology of China, Chengdu 611731 Sichuan, China 